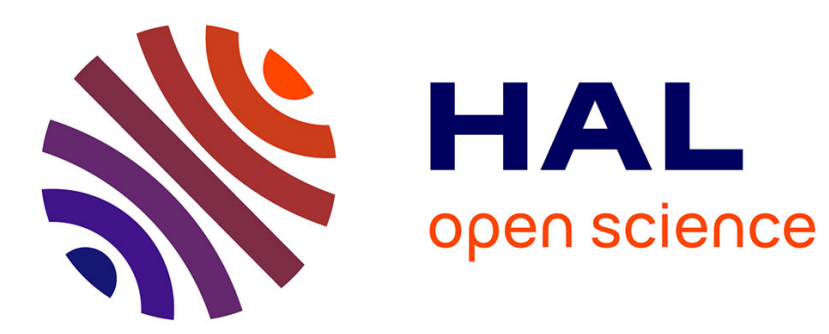

\title{
Thermal convection in the porous methane-soaked regolith of Titan: Investigation of stability
}

\author{
Leszek Czechowski, Konrad Kossacki
}

\section{To cite this version:}

Leszek Czechowski, Konrad Kossacki. Thermal convection in the porous methane-soaked regolith of Titan: Investigation of stability. Icarus, 2009, 202 (2), pp.599. 10.1016/j.icarus.2009.02.032 . hal00554486

\section{HAL Id: hal-00554486 \\ https://hal.science/hal-00554486}

Submitted on 11 Jan 2011

HAL is a multi-disciplinary open access archive for the deposit and dissemination of scientific research documents, whether they are published or not. The documents may come from teaching and research institutions in France or abroad, or from public or private research centers.
L'archive ouverte pluridisciplinaire HAL, est destinée au dépôt et à la diffusion de documents scientifiques de niveau recherche, publiés ou non, émanant des établissements d'enseignement et de recherche français ou étrangers, des laboratoires publics ou privés. 


\section{Accepted Manuscript}

Thermal convection in the porous methane-soaked regolith of Titan: Investigation of stability

Leszek Czechowski, Konrad Kossacki

PII: S0019-1035(09)00112-2

DOI: $\quad$ 10.1016/j.icarus.2009.02.032

Reference: $\quad$ YICAR 8956

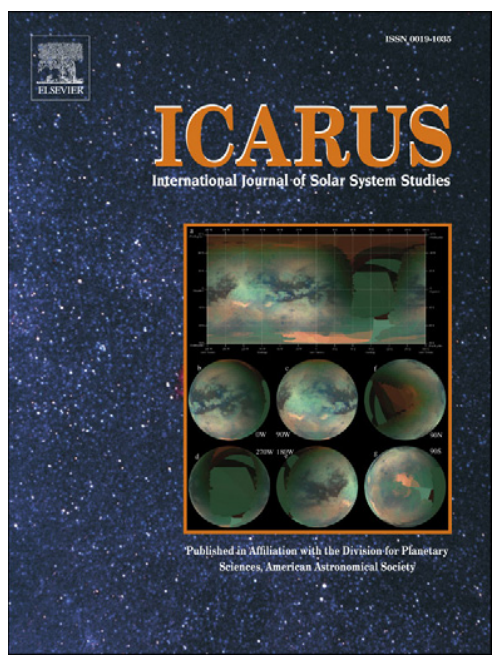

To appear in: Icarus

Received date: 9 July 2008

Revised date: 22 December 2008

Accepted date: 12 February 2009

Please cite this article as: L. Czechowski, K. Kossacki, Thermal convection in the porous methane-soaked regolith of Titan: Investigation of stability, Icarus (2009), doi:

10.1016/j.icarus.2009.02.032

This is a PDF file of an unedited manuscript that has been accepted for publication. As a service to our customers we are providing this early version of the manuscript. The manuscript will undergo copyediting, typesetting, and review of the resulting proof before it is published in its final form. Please note that during the production process errors may be discovered which could affect the content, and all legal disclaimers that apply to the journal pertain. 
Thermal convection in the porous methane-soaked regolith of Titan:

investigation of stability

Leszek Czechowski ${ }^{\text {a, }}$, Konrad Kossacki ${ }^{\text {a }}$

${ }^{\text {a }}$ Institute of Geophysics of Warsaw University, Pasteura 7, 02-093 Warszawa, Poland

Tel. +48225546834 Fax +48225546882

${ }^{*}$ Corresponding Author E-mail address: 1czech@,fuw.edu.pl

Pages 35

Tables 3

Figures 8 
Proposed running Head : Convection in regolith

\section{Editorial correspondence to:}

Dr hab. Leszek Czechowski

Institute of Geophysics of Warsaw University,

Pasteura 7, 02-093 Warszawa, Poland

Phone: (48) (22) 5546850

Fax (48) (22) 5546882

E-mail address: 1czech@,fuw.edu.pl or 1czecho@op.p1 


\begin{abstract}
Titan is the only body, other than the Earth where liquid is present on the surface. In the present work we consider behavior of methane in the pores of Titan's regolith. Using numerical model we investigate quantitative conditions necessary for the onset of convection. We have found that the methane convection in Titan's regolith is possible. It can be expected in regions where the regolith has sufficiently high porosity, independently of the geothermal heat flux.
\end{abstract}

Key Words: Titan, regolith; convection, in porous media 


\section{Introduction}

Titan is rather unusual celestial body. It is the only one in the Solar System (with the exception of Earth), where the $p$ - $T$ conditions allow an active hydrological cycle (based on hydrocarbons), as well as permanent presence of liquid on its surface. Existence of the methane ocean or lakes on Titan was suggested a decade ago, based on photochemical considerations (e.g. Stevenson and Potter, 1986; Raulin, 1987). The present atmospheric stock of methane should have been depleted by photolysis in $10^{7}$ years if it was not replenished (e.g. Lunine, 1993; Tobie et al. 2006 a). Thus, the present existence of methane suggests a possible surface (or near-surface) reservoir of methane. Further, the dominant photochemical product, ethane, is liquid at the surface $p-T$ conditions. Young et al. (1984) suggested that the methane photolysis over the age of the Solar System should lead to a layer of ethane $600 \mathrm{~m}$ thick on average (see also Tokano, 2005). This would imply the presence of an ethane-rich ocean. The presence of methane just beneath the surface, in the porous regolith, was also considered Stevenson (1992), Kossacki and Lorenz (1996). Recently, observations confirm that some liquid methane is present on the surface of Titan. The Huygens Probe detected features that indicate the presence of some liquid on the Titan's surface: methane clouds and high surface relative humidity in the vicinity of its landing site. Of course, the term 'humidity' is used here to describe the methane and/or ethane vapor in Titan's atmosphere. The presence of liquid methane is indicated also by the results of GCMS (gas chromatograph mass spectrometer) and by good cooling properties of the regolith (e.g. Tomasko et al., 2005; Lorenz et al., 2006 a). Those observations are sufficient to expect the presence of surface, or sub-surface sources of methane that replenish this gas against photo- and charged-particle chemical loss (Atreya et al., 2006). In addition, reservoirs of liquid at the surface are indicated by radar observations. Imaging failed to find evidence of a global ocean, although radar observations provided definitive evidence for the presence of lakes (Stofan et al., 2007; Hayes et al., 2008; Brown et 
al., 2008). Therefore, in some regions, Titan's regolith is probably filled with liquid methane and/or ethane. The Cassini Orbiter remote sensing shows also dry and even desert-like landscapes with dunes (Lorenz et al., 2006 b). More recently the properties of the Titan surface were described by Lunine et al. (2008) and Paganelli et al. (2008). Taking into account the diversity of the Titan landscapes we expect, that the regolith can be, in some regions, free of any liquid, or dry to a large depth. However, the presence of lakes suggests small depth to the liquid table (at least in some regions) at high latitudes in the northern hemisphere. Mitri et al. (2007) argue that high relative humidity of methane in Titan's lower atmosphere could be maintained by evaporation from lakes. They state that lakes covering $2 \%$ of the whole surface are sufficient to maintain the observed amount of atmospheric methane. This may indicate that gas exchange between the subsurface methane reservoirs and the atmosphere is slow, possibly due to the presence of some dry material with low gas permeability above the liquid table. It should be noted, that the area of lakes calculated by Mitri et al. (2007) is just a lower estimate. Probably the lakes occupy more than $2 \%$ of the Titan's surface. The processes of evaporation and subsequent precipitation lead to global transport of hydrocarbons. Note also that the lakes are probably filled with some mixtures of ethane, methane, and nitrogen rather than pure methane. The total area of lakes would have to be larger than for pure methane lakes to account for the present concentration of methane in the atmosphere.

In the present paper we investigate the possibility of thermal convection in the Titan's regolith. We assume that the regolith is saturated with liquid methane and heated from below by the geothermal heat sources. In such physical situation convection of the liquid can be expected. We attempt to determine the conditions necessary for the onset of convection. Usually convection is a more efficient mechanism of heat transfer than conduction; therefore the presence of convection should lead to a substantial change of heat flow and/or temperature 
distribution in the regolith. Moreover, the convection could dramatically redistribute the heat flow leading to origin of some specific surface structures like geysers. Different types of convection in the Titan's interior were already discussed (e.g. Tobie et. al., 2006 b). Different models of the Titan's volcanism were also presented (e.g. Fortes and Grindrod, 2005; Fortes et al., 2006; Alekseev, 2008). However, to our best knowledge our current work is the first dealing with convection in the shallow near-surface layer of the Titan's regolith.

The paper is organized as follows: In Section 2 we describe the numerical model. Section 3 contains the results of the stability investigations. In Section 4 we discuss application of our calculations to Titan. The conclusions are in the last section.

\section{Numerical model of convection in regolith}

\subsection{Physical situation}

We consider a horizontal layer of porous regolith soaked with a liquid - Fig. 1.

[Figure 1] [Table 1] Let $h$ be the thickness of the layer, and $L$ its length. Let also grad $p$ be the pressure gradient. This gradient drives the flow of the liquid given by the Darcy's formula: $\boldsymbol{v}=-(k / \eta) \operatorname{grad} p$, where $\eta$ is the dynamical viscosity of the liquid $[\mathrm{Pa} \mathrm{s}], k\left[\mathrm{~m}^{2}\right]$ is the permeability and $v\left[\mathrm{~m} \mathrm{~s}^{-1}\right]$ is a volumetric flow rate, i.e. velocity averaged over some volume (the volume must be large comparing to size of pores) (e.g. Turcotte and Schubert, 2001, Ch. 9). Note that $\boldsymbol{v}$ is not the true velocity of the fluid inside the pores. Due to very limited knowledge about Titan's regolith we describe the dependence of permeability $k$ on depth using the simple formula:

$$
k(y)=k_{0} \exp \left((y-0.5 h) / H_{p}\right)
$$

where $H_{p}[\mathrm{~m}]$ is a constant 'depth scale' and $k_{0}$ is the permeability in the middle of the considered layer (i.e. for $y=h / 2$ ). Eq. (1) approximately reproduces the results of numerical simulations dealing with the self-compaction of the regolith on Titan (Kossacki and Lorenz, 
1996). The temperature at the upper boundary is $T_{0}$. At the lower boundary we consider two alternative boundary conditions (B.C.): temperature $T_{l}=T_{0}+\Delta T$ or the density of the heat flux $f_{1}\left[\mathrm{~W} \mathrm{~m} \mathrm{~m}^{-2}\right]$. The first condition corresponds to the situation where below the regolith is a body with constant temperature (e.g. a cryomagma chamber). The second condition means the presence of a constant geothermal heat flux from below, the condition probably more appropriate for most regions of Titan. The lateral boundaries are assumed to be adiabatic (i.e., no heat flow):

$$
\left.\frac{\partial T}{\partial x}\right|_{\text {for } x=0 \text { and } x=d}=0, \quad 0<y<h
$$

\subsection{Equations}

We use the 2D numerical model based on the following system of equations: (i) the equations of motion for fluid in a porous medium, (ii) the equation of thermal conductivity, (iii) the equation of continuity and (iv) the equation of state. We assume that the liquid could be treated as incompressible so the equation of continuity and the equation of state are reduced to:

$$
\nabla \cdot \boldsymbol{v}=0
$$

and

$$
\rho_{f}=\rho_{0 f}\left(1-\alpha\left(T-T_{0}\right)\right)
$$

where $v, T, T_{0}, \rho_{f}, \rho_{0 f}, \alpha$, denote the average velocity of the fluid, temperature, reference temperature (i.e. the surface temperature), density of the fluid, reference density of the fluid, and coefficient of thermal expansion of the fluid, respectively. The equations of motion express the fact that the velocity is proportional to the pressure gradient (e.g. Turcotte and Schubert, 2001, Ch. 9) i.e.

$$
\mathrm{v}_{1}=-\frac{1}{\delta} \frac{\partial p}{\partial x}
$$




$$
\mathbf{v}_{2}=-\frac{1}{\delta}\left(\frac{\partial p}{\partial y}+\rho_{f} \alpha_{f} g\left(T-T_{0}\right)\right)
$$

where $\delta=\eta / k$ is the ratio of the viscosity $\eta[\mathrm{Pa} \mathrm{s}]$ and the permeability $k\left[\mathrm{~m}^{2}\right]$ of the matrix (the regolith). It should be noted, that the matrix does not move. The symbol $g\left[\mathrm{~m} \mathrm{~s}^{-2}\right]$ denotes the gravitational acceleration. If $\delta_{0}=\eta / k_{0}$ then Eq. (1) gives:

$$
\delta(y)=\delta_{0} \exp \left(-(y-0.5 h) / H_{p}\right)
$$

For a $2 \mathrm{D}$ flow the velocity could be expressed by the stream function $\varphi$ in the following way:

$$
\mathrm{v}_{1}=\left(\frac{\partial \varphi}{\partial y}\right), \quad \mathrm{v}_{2}=-\left(\frac{\partial \varphi}{\partial x}\right)
$$

Now, the equation of continuity (3) is satisfied automatically (e.g. Czechowski 1993; Schubert et al. 2001). After differentiating Eq. (5) with respect of $y$ and Eq. (6) with respect of $x$ and subtracting them we get:

$$
\left(\frac{\partial}{\partial y}\left(\delta \frac{\partial \varphi}{\partial y}\right)+\frac{\partial}{\partial x}\left(\delta \frac{\partial \varphi}{\partial x}\right)\right)=\rho_{f} \alpha g\left(\frac{\partial T}{\partial x}\right)
$$

Equation of heat transfer is (e.g. Turcotte and Schubert, 2001, Ch. 9):

$$
\left(\frac{\partial T}{\partial t}\right)+C\left(\mathrm{v}_{1} \frac{\partial T}{\partial x}+\mathrm{v}_{2} \frac{\partial T}{\partial y}\right)=\kappa_{m}\left(\frac{\partial^{2} T}{\partial x^{2}}+\frac{\partial^{2} T}{\partial y^{2}}\right),
$$

where $t$ is time, $\kappa_{\mathrm{m}}=\lambda_{\mathrm{m}} / \rho_{\mathrm{m}} c_{\mathrm{m}}$ is a thermal diffusivity, and $C$ is a dimensionless parameter given by:

$$
C=\left(\frac{\rho_{0 f} c_{f}}{\rho_{m} c_{m}}\right),
$$

The $\lambda_{\mathrm{m}}, \rho_{\mathrm{m}}, c_{\mathrm{m}}$ denote the coefficient of thermal conductivity, density, and specific heat of the matrix (dry regolith). The $c_{f}$ denotes specific heat of the fluid.

\subsection{Dimensionless form of equations}


Let us define natural units of time, length, velocity, and stream function for our problem as follow:

$$
\tau=h^{2} / \kappa_{m}, \quad d=h, \quad \omega=\kappa_{m} / h, \quad \Phi=\kappa_{m} .
$$

For isothermal lower boundary the natural unit of temperature is given by the formula: $\Theta=(T$ $\left.-T_{0}\right) / \Delta T$. If heat flow density for the lower boundary is given then $\Theta=\lambda_{\mathrm{m}}\left(T-T_{0}\right) /\left(f_{l} d\right)$.

The system of equations (8)-(9) could be transformed into dimensionless form by means of the following substitutions (primed variables are given in the natural units)

$$
t=t^{\prime} \tau, \quad x=x^{\prime} d, \quad \mathbf{v}=\mathbf{v}^{\prime} \omega, \quad T=T^{\prime} \Theta+T_{0} .
$$

Here, the temperature $T_{0}$ is the reference temperature corresponding to the surface temperature. Transformation (12) reduces the number of the system parameters to 2 dimensionless numbers: the Rayleigh number $R a$ and the number $C$. The $C$ is given by (10) while the Rayleigh number $R a$ is given by:

$$
\begin{aligned}
& R a=\frac{\rho_{f} g \alpha h \Delta T}{\delta_{0} \kappa_{m}} \text { for temperature } T_{l} \text { given at the lower boundary, or } \\
& R a=\frac{\rho_{f} g \alpha h^{2} f}{\delta_{0} \lambda_{m} \kappa_{m}} \text { for heat flow density } f_{l} \text { given at the lower boundary. }
\end{aligned}
$$

Finally, we obtain the following dimensionless equations:

$$
\begin{aligned}
& \left(\frac{\partial}{\partial y^{\prime}}\left(\delta^{\prime} \frac{\partial \varphi^{\prime}}{\partial y^{\prime}}\right)+\frac{\partial}{\partial x^{\prime}}\left(\delta^{\prime} \frac{\partial \varphi^{\prime}}{\partial x^{\prime}}\right)\right)=R a\left(\frac{\partial T^{\prime}}{\partial x^{\prime}}\right), \\
& \left(\frac{\partial T^{\prime}}{\partial t^{\prime}}\right)+C\left(\mathrm{v}_{1}^{\prime} \frac{\partial T^{\prime}}{\partial x^{\prime}}+\mathrm{v}_{2}^{\prime} \frac{\partial T^{\prime}}{\partial y^{\prime}}\right)=\left(\frac{\partial^{2} T^{\prime}}{\partial x^{\prime 2}}+\frac{\partial^{2} T^{\prime}}{\partial y^{\prime 2}}\right),
\end{aligned}
$$

where $\delta^{\prime}=\delta / \delta_{0}$ is a dimensionless function:

$$
\delta^{\prime}\left(y^{\prime}\right)=\exp \left(-\left(y^{\prime}-0.5\right) / H_{p}^{\prime}\right)
$$

where $H_{p}^{\prime}=H_{p} / h$. If steady state convection is assumed then the time derivative in the equation of the heat transfer (Eq. 16) is equal to zero.

Dimensionless boundary conditions are: 
at the upper boundary (isothermal): $T^{\prime}=0$ for $y^{\prime}=1$, at the lower boundary (if isothermal): $T^{\prime}=1$ for $y^{\prime}=0$, or: at the lower boundary (if heat flow density given): $\partial T^{\prime} / \partial x^{\prime}=1$ for $y^{\prime}=0$. Adiabatic boundary conditions $\partial T^{\prime} / \partial x^{\prime}=0$ are assumed at the lateral boundaries i.e. at $x^{\prime}=0$ and $x^{\prime}=L^{\prime}$ where $L^{\prime}=L / h$. Eventually our problem contains four dimensionless numbers: the Rayleigh number $R a$, the dimensionless number $C$, the depth scale $H_{\mathrm{p}}$, and the aspect ratio (i.e. dimensionless length of the regolith layer - Fig. 1) $L$ '.

\subsection{Numerical method}

The system of equations (15)-(16) is solved using a numerical code developed by L. Czechowski. The code uses the finite difference method (FDM). The Cartesian system of coordinates is used for discretization. The equations (15)-(16) are solved in a rectangular region $\left[0, L^{\prime}\right] \times[0,1]$. An upstream, conservative scheme is used to approximate the advective term (i.e. the term $\boldsymbol{v} \cdot \nabla$ ) in the energy equation (16). A combination of methods is used to solve the resulting algebraic equations: Chebyshev iterations and the minimum residua method with iterative refinement. Typical resolution is $1 / 32$, so the grid size is $32 \times 32$ cells for a square region, but in some cases a higher resolution is applied (1/64). The model is tested in usual ways. The numerical solutions were compared with analytical solutions (see the next section). Moreover, unphysical artificial analytical solutions were constructed for comparison (for more details see e.g. Czechowski, 1993). Convergence tests are performed by running the

same models while decreasing the grid size. Another test included different perturbations of the initial distribution of temperature.

\section{Investigation of stability}


Instability of the system, i.e. the onset of convection, means that any infinitesimal disturbance increases eventually leading to a finite motion. Let us assume that the B.C. at the upper boundary (i.e. the temperature $T_{0}$ ) and the B.C. at the lower boundary (i.e. the temperature $T_{l}$ or the heat flow $f_{l}$ ) are independent of $x$. In such symmetrical situation convection starts only if the Rayleigh number $R a$ exceeds some critical value $R a_{\mathrm{cr}}$. For the simple case of an infinite layer (i.e. $L^{\prime}=\infty$ ) with constant permeability (i.e. $H_{p}{ }^{\prime}=\infty$ ), and for the same thermal properties of the fluid and the matrix (i.e. for $C=1$ ) the stability condition could be found analytically (e.g. Turcotte and Schubert, 2001, Ch. 9). It is found that infinitesimal sinusoidal disturbances with the dimensionless wavelength $l$ grow if $R a>R a^{0}$ where:

$$
R a^{0}=\frac{\left[\left(\frac{2 \pi}{\ell}\right)^{2}+\pi^{2}\right]^{2}}{\left(\frac{2 \pi}{\ell}\right)^{2}}
$$

A real disturbance would contain any wavelength $l$, so convection is inhibited only if $R a<R a^{0}$ for every $l$. The minimum of $R a^{0}(l)$ is known as the critical value of the Rayleigh number $R a_{\mathrm{cr}}$. For the case described by Eq. (18) $R a_{\mathrm{cr}}$ is:

$$
R a_{\mathrm{cr}}=\min R a^{0}(l)=4 \pi^{2}=39.4784,
$$

and it is reached for $l=2$.

For testing purposes we investigated the above stability problem using our numerical model. We used small sinusoidal disturbances. The comparisons of numerical and analytical data are given in Fig. 2. The results show very good agreement so our approach appears to be correct.

\section{[Figure 2]}

In the present paper we investigate the stability of the system for several values of the dimensionless numbers characterising our problem, i.e. for $C, H_{\mathrm{p}}$, and $L^{\prime}$. We used disturbances containing all possible wavelength $l$, so we do not investigate $R a^{0}$ but $R a_{\mathrm{cr}}$ only. 
Stability of the system (decreasing disturbances) means that $R a$ is lower than $R a_{\mathrm{cr}}$, the instability (growing disturbances) means that $R a$ is higher than $R a_{\mathrm{cr}}$. Note also that $R a^{0}$ has no practical significance (it is important for theoretical considerations only).

The results of the stability investigations are presented in Figs. 3-7. We find that $R a_{\mathrm{cr}}$ decreases strongly with increasing $C$ - Figs. 3 and 4. This fact is not unexpected because higher $C$ means larger role of liquid in the heat transport. Therefore an increase of $C$ means more unstable situation and consequently a decrease of the critical value of $R a_{c r}$.

\section{[Figure 3, Figure 4]}

The critical value $R a_{\text {cr }}$ depends also on $H_{\mathrm{p}}$ and $L^{\prime}$. However, these relations are weak or moderate. Let us consider $R a_{\mathrm{cr}}$ as a function of $H_{\mathrm{p}}$ (Figs. 5 and 6). Note that large $H_{\mathrm{p}}$ means low gradient of permeability. The case of constant permeability corresponds to $H_{\mathrm{p}}$ being infinite. The case of low $H_{\mathrm{p}}$ means high gradient of permeability. We choose such function $k(y)$ that the dimensionless permeability at the centre of the layer is the same $k^{\prime}=k / k_{0}=1$ for any $H_{\mathrm{p}}$. Consequently, a decrease of $H_{\mathrm{p}}$ implies an increase of permeability above $y=h / 2$ and corresponding decrease of $k$ below $y=h / 2$. Eventually, both effects of changing $H_{\mathrm{p}}$ are partially compensated. Therefore, for most of the investigated range of $H_{\mathrm{p}}$ the dependence of $R a$ on $H_{\mathrm{p}}$ is rather weak. Only for very small $H_{\mathrm{p}}\left(H_{\mathrm{p}}<0.3\right)$ we can see substantial decrease of $R a_{\mathrm{cr}}$ resulting from high permeability in the upper part of the layer - Figs. 5 and 6.

[Figure 5, Figure 6, Figure 7]

Figure 7 presents $R a_{\mathrm{cr}}$ as a function of the dimensionless length $L^{\prime}$. The dependence (a weak one) of the $R a$ is a result of difficulty in optimal fitting of the number and the size of convective cells into a finite container. For 'inconvenient' $L$ ' the $R a_{\mathrm{cr}}$ is higher (compare an increase of $R a_{\mathrm{cr}}$ for $\left.L^{\prime}<1\right)$.

\section{Application to Titan}




\subsection{Observations}

In this work we investigate heat and fluid transport within the porous regolith on Titan. According to Brown et al. (2008) Ontario Lacus contains some mixture of ethane, methane, nitrogen and other low-molecular-mass hydrocarbons. One can assume that the same liquid is present in the regolith beneath the surface. We attempt to answer the question, whether the liquid filling the pores can be in a convective motion. If convection operates then the heat transport through the regolith is much more efficient than under static conditions.

In our model pores are filled with liquid up to the surface. This is not a critical assumption. Similar results are obtained for slightly different upper boundary conditions, i.e. for a layer of regolith with dry upper part and lower part soaked with liquid. In reality the depth to the liquid table is probably non-uniform. Observations of the Titan's topography performed by the Cassini spacecraft reveal the presence of the large dune fields (a desert) in the equatorial region (Lorenz et al. 2006 b). In this region the liquid table is probably deep. However, hydrocarbon lakes are observed in the North Polar Region (Stofan et al. 2007; Hayes et al. 2008). This suggests that the regolith is regionally soaked with liquid hydrocarbons up to the surface and in some regions the table may be above the surface. Lunine et al. (2008) presented observations indicating the presence of liquids also in the South Polar Region. The authors observed in the southern hemisphere progressive poleward descent from a high, dissected, and partly hilly terrain down to a low flat plain with embayment and deposits suggestive of the past or even current presence of hydrocarbon liquids. The above observations make both polar regions good candidates for the methane convection in the near-surface regolith layer. However, other regions could be considered as well.

\subsection{Range of dimensionless parameters $L^{\prime}, H_{\mathrm{p}}$ and $C$}


Figures 3-7 give values of $R a_{c r}$ as a function of the dimensionless parameters: $L$ ', $H_{\mathrm{p}}$ and $C$. The parameter $L$ ' depends on the geometry. On the one hand, it could be low for regolith filling narrow crack between compact and impenetrable walls. On the other hand $L$ ' could be very large for vast lowland covered by a uniform layer of regolith. The value of the 'depth scale' $H_{\mathrm{p}}$ also could vary in a wide range, depending on the properties of the regolith. However, the Rayleigh number $R a$ does not significantly depend either on $L$ ' or on $H_{\mathrm{p}}$.

$R a$ depends strongly on the coefficient $C$, especially for $C<0.5$. The value of $C$ depends on the density and thermal properties of the regolith and the liquid (see Eq. 10). The regolith on Titan is commonly considered as a porous mixture of water ice, other sorts of ices, tholins, and clathrates (e.g. Waite et al., 2007; Fortes and Stofan, 2005; Osegovic and Max, 2005). The exact composition and structure of the regolith remain unknown. 'Titan tholin' is a nitrogen-rich organic substance produced by the irradiation of gaseous mixtures of nitrogen and methane. The orange-red haze of Titan's atmosphere is probably caused by the presence of tholins. Noteworthy is also the 'ice tholin', which is formed by the irradiation of clathrates of water and organic compounds. The regolith can also contain some silicates. Although the interior of Titan is differentiated (i.e. silicates are concentrated in the core - Tobie et al., 2005; Sotin and Tobie, 2008) but the silicates could be of meteoroid origin and/or part of the crust could be not included in the differentiation.

We do not know the exact composition of the liquid (see the note above). Here we discuss methane but our results could also be applied to ethane or any other liquid because our calculations cover some range of dimensionless parameters.

\section{[Table 2]}

For the calculation of $C$ we use the known properties of silicate rocks, water ice, and methane - Table 2. The method of calculation of the specific heat for a mixture of ice and silicates is taken from Kossacki and Leliwa-Kopystynski (1993). For a regolith composed of 
silicates only we obtain $C=0.46$. For regolith composed of $70 \%$ silicate and $30 \%$ of pure water ice (it correspond to the average composition of Titan) we get $C=0.83$. For pure water ice regolith $C=1.88$. In conclusion we should consider $C$ in some range above and below 1 . The range $C>1.5$ is somewhat less important because the dependence $R a_{c r}$ on $C$ is weaker than for lower $C$ (compare Figs. 3 and 4). Note also the possibility of stratification of liquid in the regolith resulting from different densities of methane and ethane. To overcome this stratification the critical value of the Rayleigh number has to be higher.

\subsection{Range of $R a$ for Titan}

Convection starts if the true value of $R a$ exceeds $R a_{c r}$. The values of $R a_{c r}$ are presented in Figs. 3-7. Let us consider now true values of $R a$. They depend on many parameters - Eqs. (13)-(14). Some of the parameters could vary only in rather narrow ranges (e.g. $g, \alpha$ ) while others could vary in very wide ranges, especially the reference permeability $k_{0}$ and the thickness $h$.

Let us consider $h$. Kossacki and Lorenz (1996) simulated the process of self-compaction of a partially soaked regolith composed of water ice, or a mixture of water ice and sand. In all cases the regolith quickly settled down to a near-equilibrium. The final profiles of porosity $\psi$ versus depth appeared to be almost independent of the initial thickness of the regolith. Assuming the surface porosity 0.5 they obtain porosity 0.475 at $1 \mathrm{~km}$ depth, 0.36 at $2 \mathrm{~km}$, 0.26 at $3 \mathrm{~km}$, and 0.22 at $4 \mathrm{~km}$. The convective motion of liquid filling the pores requires continuous paths connecting top and bottom of the layer (i.e. percolation). The percolation threshold is defined as $1-\psi_{\mathrm{p}}$ where $\psi_{\mathrm{p}}$ is the porosity for which percolation appears. The $\psi_{\mathrm{p}}$ depends on the packing geometry of the medium and on the grain shape of the regolith. For a fractal medium composed of cubes $\psi_{\mathrm{p}}$ is about 0.7 (Shoshany et al., 2002). However, in a simple cubic matrix of equal spherical grains flow paths exist (the medium is permeable) even 
for the porosity as low as 0.5 . If the grains are flattened at the contact points then $\psi_{\mathrm{p}}<0.5$. For the purpose of the current analysis we assume $\psi_{\mathrm{p}}=0.3$. Thus, the upper limit of the thickness $h$ in the present paper is $2.5 \mathrm{~km}$.

\section{[Table 3] [Figure 8]}

Table 3 presents $R a$ calculated for different values of $k_{0}$ and $h$. The heat flow density and the temperature gradient are calculated assuming only radiogenic heat production (no tidal heating is included) and thermal conductivity. The radioactive elements are contained only in the silicate component of the satellite. Chondritic heat production is assumed (Schubert et al. 1986). Comparing $R a$ from Table 3 and $R a_{c r}$ given by Figs. 3 - 7 one can conclude that the convection in the regolith is possible even for moderate thickness of the regolith (e.g. $10 \mathrm{~m}$ ) and moderate permeability (e.g. $10^{-7} \mathrm{~m}^{2}$ ). For high permeability (e.g. $10^{-6}$ $\mathrm{m}^{2}$ ) convection is possible even for a thin $(h=1 \mathrm{~m})$ regolith layer (note that $R a>77-$ Table 3 ). For a thick layer, e.g. $h=1000 \mathrm{~m}$, convection is possible for low permeability corresponding to terrestrial sandstone $\left(10^{-14} \mathrm{~m}^{2}\right)$.

Unfortunately, we do not know the thickness of the Titan's regolith, nor its permeability. The mentioned calculations of Kossacki and Lorenz (1996) give the upper limit only. Nevertheless, our current calculations suggest that convection in regolith is a common process in regions where the regolith is soaked with methane or other liquids.

Convection is an effective way of heat transfer. If the heat flow is given at the base of the regolith then convection reduces the temperature gradient in the layer compared to the situation without convection. Our calculation of finite convection indicates that for $R a=10^{3}$ the averaged temperature gradient could be reduced down to $15 \%$ (i.e. 6 - 7 times) of the gradient without convection. For isothermal lower BC, i.e. when temperature $T_{l}$ is given, convection significantly increases the heat flow at the surface. Concluding: our result suggests 
that thermal models of Titan regolith should take into account convection in the regolith unless the regolith is dry.

\subsection{Convection in volcanic regions and terrestrial phenomena of similar origin}

Terrestrial sediments on the terrestrial continent (e.g. post-glacial layer of sand and stones) are often a few dozen meters thick and are soaked with water. However the water temperature (at least in moderate latitudes) is close to $4^{\circ} \mathrm{C}$, i.e., the coefficient of thermal expansion is low and consequently $R a$ is low. For temperatures in the range $0{ }^{\circ} \mathrm{C}-4^{\circ} \mathrm{C}$ the coefficient is even negative thus inhibiting convection. Hydrothermal convection is important in the region of high geothermal heat flow. The most powerful hydrothermal activity is observed in the spreading centers where new oceanic floor is created (e.g. Turcotte and Schubert, 2001, Ch. 1). On land the hydrothermal activity is observed in volcanic regions (and other high heat flow regions) and takes form of geysers, hot springs, and mud volcanoes (e.g. Kopf, 2002; Revil, 2002; Henry et al., 1996). The circulating water often dissolves minerals in one place and deposits them in another place (e.g. Carson and Screaton, 1998). Many of these processes could have analogies on Titan.

The values of $R a$ presented in Table 3 and Fig. 8 are calculated using average density of heat flow $f_{l}$. However, the heat flow distribution is not uniform. According to Lopes et al. (2007) large regions of possible cryo-volcanic activity are observed on Titan. The origin and role of the cryo-volcanism is considered by Fortes et al. (2006). In volcanic regions the heat flow density could be significantly higher then the average flow on the satellite. Locally (e.g. on volcanic domes) the heat flow could be a few orders of magnitude higher than average. The Rayleigh number is proportional to the heat flow (see Eq. (14)) so also $R a$ could be locally enhanced by orders of magnitude. This implies very intensive convection. One can expect hot springs, geysers and some other forms of geothermal activity. The existence of 
mud volcanoes on Titan was also suggested (Alekseev, 2008; Fortes and Grindrod, 2005). The fluids circulating in pores could lead to closing of pores (as a result of deposition) or to their enlargement (as a result of solubility) - Eluszkiewicz and Stevenson, 1990; Raulin, 1987. If some structures mentioned by the cited authors (Fortes and Grindrod, 2005; Lopes et al. 2007; and Alekseev, 2008) are indeed signatures of convection, their size can be used to deduce the intensity of convection and the size of convective cells.

Let us also stress that in Table 2 we included radiogenic heat only. It is possible that tidal heating is comparable (or even higher) to the radiogenic one. The main differences between these two modes of heating are:

(i) There is no orientation of the homogenous radiogenic heating while tidal heating gives the pattern strongly oriented in respect to the planet-satellite line (e.g. Czechowski and Leliwa-Kopystynski 2005).

(ii) The rate of radiogenic heating is determined by the content of radioactive elements and is decreasing with time. The tidal heating has no such limitations, it could be weak or intensive depending on the satellite's orbit.

(iii) If tidal heating is substantial then the total heat flux is significantly higher, making the onset of convection easier. Note, however, that the tidal interactions do not result in tidal motion similar to the tides in the terrestrial oceans. The tidal effects are negligible for small Titan's lakes.

\section{Conclusions and future work}

In the present paper we investigated quantitatively the possibility of the methane convection in the porous regolith of Titan. We found that: 
(i) Critical value of the Rayleigh number depends mainly on the parameter $C$ given by Eq. (10). For $C=0.4$ the critical value of $R a$ is approximately 100 , while for $C=1 R a_{c r}$ is two times lower (Figs 3 and 4).

(ii) Convection in the Titans regolith is possible even for moderate permeability and moderate thickness of the regolith layer Table 3 and Fig. 8).

(iii) Convection leads to the significant modifications of the thermal conditions in the regolith. Upward convective currents should increase the temperature of the sub-surface layer while downward currents could decrease its temperature. We do not know whether some observed temperature differences are of such origin (Mitri et al. 2007; Paganelli et al. 2007). It should be noted, that the cited brightness temperature contrasts are derived from the passive radar data. Hence, they correlate with the physical temperature contrasts only if the surface is flat and uniform.

The next step of the presented investigations should be the calculations of the finite amplitude convection and the simulations of the time-dependent convection in the regolith. Such simulations, which should give better insight into the process, are currently being prepared. Moreover, we are looking for observational facts that could indicate the presence of convection in the Titan's regolith (e.g. Lopes et al. 2007; Paganelli et al. 2007, 2008; Radebaugh et al. 2007; McCord et al., 2008). The possibility of observational detection of the signatures of convection depends on the rate of convection (i.e. the efficiency of the convective heat transport), and on its pattern. Vigorous convection in small cells should lead to higher surface temperature contrast than that expected for slow, large-scale convection. Investigations of a possible rate and character of convection (detectability of convection) require additional simulations and are outside the scope of our current work.

\section{Acknowledgments}


This work was partially supported by the Polish Ministry of Education and Science (grants 0576/H03/2007/32, and 2P04 D05428). We are also grateful to Dr. A. Dominic Fortes from University College London and to Alexander Hayes from California Institute of Technology for their remarks and suggestions.

\section{References}

Alekseev, V.A., 2008. On the probability of cryogenic mud volcanism on Titan. Solar System Research, 42(2), 139-143, doi: 10.1007/s11208-008-2005-9.

Atreya, S.K., Adams, E.Y., Niemann, H.B., Demick-Montelara, J.E., Owen, T.C., Fulchignoni, M., Ferri, F., Wilson, E.H., 2006. Titan's methane cycle. Planet. Space Sc. $54,1177-1187$.

Brown, R.H., Soderblom, L.A., Soderblom, J.M., Clark, R.N., Jaumann, R., Barnes, J.W., Sotin, C., Buratti, B., Baines, K.H., Nicholson, P.D., 2008. The identification of liquid ethane in Titan's Ontario Lacus. Nature 454, 607-610, doi:10.1038/nature07100.

Carson, B., Screaton, E.J., 1998. Fluid flow in accretionary prisms: evidence for focused, time-variable discharge. Rev. Geophys. 36 (3), 329-351.

Czechowski, L., 1993. Theoretical approach to mantle convection. In Dynamics of the Earth's Evolution, ed. R. Teisseyre, L. Czechowski and J. Leliwa-Kopystyński, pp. 161-271, Elsevier, Amsterdam.

Czechowski, L., Leliwa-Kopystynski, J., 2005. Convection driven by tidal and radiogenic heating in medium size icy satellites. Planet. Sp. Sci. 53, 749-769.

Eluszkiewicz, J., Stevenson, D.J., 1990. Physico-chemical state of Titan's subsurface layers. LPSC XXI, 323

Fortes, D. S., Grindrod, P.M., 2005. Modelling of possible mud volcanism on Titan. Icarus, 182, 550-558, doi 10.1016/j.icarus.2005.11.013. 
Fortes, D. S., Grindrod P.M., Trickett, S.K., Vocadlo, L., 2006. Ammonium sulphate on Titan: possible origin and role in cryovolcanism. Icarus, 188, 139-153, doi: 10.1016/j.icarus.2006.11.002.

Fortes, D. S., Stofan, E.R., 2005. Clathrate formation in the near-surface environment of Titan. LPSC XXXVI, 1123.pdf.

Hayes, A.; Aharonson, O.; Callahan, P.; Elachi, C.; Gim, Y.; Kirk, R.; Lewis, K.; Lopes, R.; Lorenz, R.; Lunine, J.; and 4 coauthors, 2008. Hydrocarbon lakes on Titan: Distribution and interaction with a porous regolith. GRL, doi:10.1029/2008GL033409.

Henry, P., Le Pichon, X., Lallemant, S., Lance, S., Martin, J.B., Foucher, J-P., Fiala-Medioni, A., Rostek, F., Guilhaumou, N., Pranal, V., Castrec, M., 1996. Fluid flow in and around a mud volcano field seaward of the Barbados accretionary wedge: results from Manon cruise. J. Geophys. Res., 101 (B9), 20297-20323.

Jain S.C., Nanda, V.S., 1974. Equation of state and other associated properties of liquid argon and liquid methane. J. Phys. C: Solid St. Phys., 4, 3045-3056.

Kossacki, K.J., Lorenz, R.D., 1996. Hiding Titan's ocean: densification and hydrocarbon storage in an icy regolith. Planet. Space Sc. 44, 1029-1037

Kossacki K.J., Leliwa-Kopystynski, J. 1993. Medium-size icy satellites: thermal and structural evolution during accretion. Planet. Space Sci., 41, 729-741.

Kopf, A.J., 2002. Significance of mud volcanism. Rev. Geophys., 40 (2), 1005, doi: 10.1029/2000RG000093.

Lopes R.M.C and 43 co-authors, 2007. Cryovolcanic features on Titan's surface as revealed by the Cassini Titan Radar Mapper. Icarus 186, 395-412. 
Lorenz, R.D., Niemann, H.B. Harpold, D.,N., Way, S.H., Zarnecki, J.C., 2006 a. Titan’s damp ground: constraints on Titan surface thermal properties from the temperature evolution of the Huygens GCMS inlet. Meteoritics \& Planetary Science, 41 (11), 1695-1835.

Lorenz, R.D., and 39 colleagues, 2006 b. The sand seas of Titan: Cassini RADAR observations of longitudinal dunes. Science 312, 724-727.

Lunine, J.I., 1993. Does Titan have and Ocean? A Review of Current Understanding of Titan's Surface. Reviews of Geophysics, 31, 131-149.

Lunine, J. I.; Elachi, C.; Wall, S. D.; Janssen, M. A.; Allison, M. D.; Anderson, Y.; Boehmer, R.; Callahan, P.; Encrenaz, P.; Flamini, E.; and 34 coauthors, 2008. Titan's diverse landscapes as evidenced by Cassini RADAR's third and fourth looks at Titan. Icarus, 195, 415-433.

McCord, T.B., Hayne, P., Combe, J-P.,Hansen, G.B., Barnes, J.W., Rodriguez, S., Le Mouélic, S., Kevin, E., Baines, H., Buratti, B.J., Sotin, C., Nicholson, P., Jaumann, R., Nelson, R., and the Cassini VIMS Team, 2008. Titan's surface: Search for spectral diversity and composition using the Cassini VIMS investigation. Icarus 194, 212-242.

Mitri, G., Showman, A.P., Lunine, J.I., Lorenz, R.D., 2007. Hydrocarbon lakes on Titan. Icarus $186,385-394$.

Osegovic, J.P., and Max, M.D., 2005. Compound clathrate on Titan's surface. J. Geophys. Res., 110, E08004, doi: 10.1029/2005JE002435.

Paganelli, F., Janssen, M. A., Stiles, B., West, R., Lorenz, R. D., Lunine, J. I., Wall, S. D., Callahan, P., Lopes, R.M., Stofan, E., Kirk, R.L., Johnson, W.T.K., Roth, L., Elachi, C., the Radar Team., 2007. Titan's surface from the Cassini RADAR SAR and high resolution radiometry data of the first five flybys. Icarus 191, 211-222.

Paganelli, F., Janssen, M. A.; Lopes, R. M.; Stofan, E.; Lorenz, R. D.; Lunine, J. I.; Kirk, R. L.; Roth, L.; Elachi, C., the Cassini Radar Team, 2008. Titan's surface from the Cassini 
RADAR radiometry data during SAR mode. Planet. Space Sc., DOI :10.1016/j.pss.2007.03.015.

Radebaugh, J., Lorentz R.D., Randolph, L.K., Lunine, J. I., Stofan, E.R., Lopes, R.M.C., Wall, S.D. and Cassini Radar Team. 2007. Mountains on Titan observed by Cassini Radar. Icarus 192, 77-91.

Raulin, F., 1987. Organic chemistry in the oceans of Titan. Adv. Space Res., 7(5), 71-81.

Revil, A., 2002. Genesis of mud volcanoes in sedimentary basins: a solitary wave-based mechanism. Geophys. Res. Let., 29 (12), 1574, doi: 10.1029/2001GL014465.

Schubert, G., Spohn, T., Reynolds., R.T., 1986. Thermal histories, compositions and internal structures of the moons of the Solar System. In Satellites, 224-292, eds. J. A. Burns, M. S. Matthews, University Arizona Press, Tucson.

Schubert, G., Turcotte, D. L. Olson, P., 2001. Mantle convection in the Earth and planets. Cambridge Univ. Press, Cambridge, UK.

Shoshany, Y., Prialnik D., and Podolak M., 2002. Monte Carlo Modeling of the Thermal Conductivity of Porous Cometary Ice. Icarus, 157, 219-227.

Stevenson, D. J., and Potter, B.E., 1986. Titan's latitudinal temperature distribution and seasonal cycle. GRL, 13, 93-96.

Sotin, C., Tobie, G., 2008. Titan's hidden ocean. Science, 319 (5870), 1629-1630, doi: 10.1126/science.1155964.

Stevenson, D.J., 1992. The Interior of Titan, Symposium on Titan. ESA SP-338, 17-22.

Stofan, E.R., Elachi, C., Lunine, J.L., Lorenz, R.D., and 34 colleagues. 2007. The lakes of Titan. Nature, 445, 61-64.

Tobie, G., Lunine, J., Sotin, C., 2006 a. Episodic outgassing as the origin of atmospheric methane on Titan. Nature 440, 61-64, doi: 10.1038/nature04497. 
Tobie G., Choblet G., Sotin C., Mitri G.. Lunine J. I., and Showman A.P., 2006 b. Numerical simulation of plume cryovolcanism: implication for methane outgassing on Titan. Lunar and Planetary Science XXXVII, 2006, 1797.

Tobie G., Grasset, O., Lunine J. I., Mocquet, A., and Sotin, C., 2005. Titan's internal structure inferred from a coupled thermal-orbital model. Icarus, 175, 496-502, doi: 10.1016/j.icarus.2004.12.007.

Tokano, T. Thermal structure of putative hydrocarbon lakes on Titan. Adv. Space Res. 36, 286-294, 2005.

Tomasko, M.G., and 39 colleagues, 2005. Rain, winds and haze during the Huygens probe's descent to Titan's surface. Nature 438, 765-778.

Turcotte, D. L Schubert, G., 2001. Geodynamics, (2-nd ed.) Cambridge Univ. Press, Cambridge, UK.

Waite, Jr. J.H., Young, D.T., Cravens, T.E., Coates, A.J., Crary, F.J., Magee, B., Westlake, J., 2007. The process of tholin formation in Titan's upper atmosphere. Science, 316, 870-875.

Young, Y.L., Allen, M., and Pinto, J.P., 1984. Photochemistry of the atmosphere of Titan: comparison between models and observations. Astrophys. J. 55, 465-506. 


\section{Table 1}

\section{Notations}

\begin{tabular}{|c|c|c|}
\hline$c_{\mathrm{m}}, c_{\mathrm{f}}$ & $\mathrm{J} \mathrm{kg}^{-1} \mathrm{~K}^{-1}$ & Specific heat for matrix and fluid, respectively \\
\hline$C=\left(\rho_{0 \mathrm{f}} c_{\mathrm{f}} / \rho_{\mathrm{m}} c_{\mathrm{m}}\right)$ & 1 & Dimensionless number \\
\hline$f_{l}$ & $\mathrm{~W} \mathrm{~m}{ }^{-2}$ & Heat flow density at lower boundary \\
\hline$h$ & M & thickness of the regolith layer \\
\hline$H_{p}$ & M & Depth scale \\
\hline$g$ & $\mathrm{M} \mathrm{s}^{-2}$ & Gravity \\
\hline$k, k_{0}$ & $\mathrm{~m}^{2}$ & Permeability, reference permeability \\
\hline$p$ & $\mathrm{~Pa}$ & Pressure \\
\hline$R a$ & 1 & Rayleigh number \\
\hline$T$ & $\mathrm{~K}$ & Temperature \\
\hline$T_{0}$ & $\mathrm{~K}$ & Surface temperature, \\
\hline$T_{l}$ & $\mathrm{~K}$ & Temperature at the lower boundary \\
\hline$t$ & $\mathrm{~s}$ & Time \\
\hline$V=\left(V_{1}, V_{2}\right)$ & $\mathrm{m} \mathrm{s}^{-1}$ & Velocity vector \\
\hline$x$ & $\mathrm{~m}$ & $\mathrm{x}$ - coordinate \\
\hline y & $\mathrm{m}$ & $\mathrm{y}$-coordinate \\
\hline$\alpha$ & $\mathrm{K}^{-1}$ & Coefficient of thermal volume expansion of fluid \\
\hline$\delta=\eta / k$ & Pa s m ${ }^{-2}$ & ratio of the viscosity $\eta$ and the permeability $k$ \\
\hline$\Delta T$ & $\mathrm{~K}$ & Temperature difference \\
\hline$\eta$ & $\mathrm{Pa} \mathrm{s}$ & Viscosity \\
\hline$\Theta=\left(T-T_{0}\right) / \Delta T$ & $\mathrm{~K}$ & Natural temperature unit \\
\hline$\kappa_{\mathrm{m}}=\lambda_{\mathrm{m}} /\left(\rho_{\mathrm{m}} c_{\mathrm{m}}\right)$ & $\mathrm{m}^{2} \mathrm{~s}^{-1}$ & Temperature diffusivity of matrix \\
\hline & $\mathrm{J} \mathrm{m}^{-1} \mathrm{~s}$ & Thermal conductivity of matrix \\
\hline$\rho, \rho_{0}$ & $\operatorname{kg} \mathrm{n}$ & Density, reference density \\
\hline$\tau=h^{2} / \kappa_{m}$ & & Natural time unit \\
\hline$\varphi$ & $\mathrm{m}^{2} \mathrm{~s}$ & Stream function \\
\hline$\Phi=\kappa_{m}$ & & Natural stream function unit \\
\hline$\omega=\kappa_{m} / h$ & $\mathrm{~m} \mathrm{~s}^{-1}$ & Natural velocity unit \\
\hline
\end{tabular}




\section{Table 2}

\section{Data about Titan and properties of regolith and methane used for calculations}

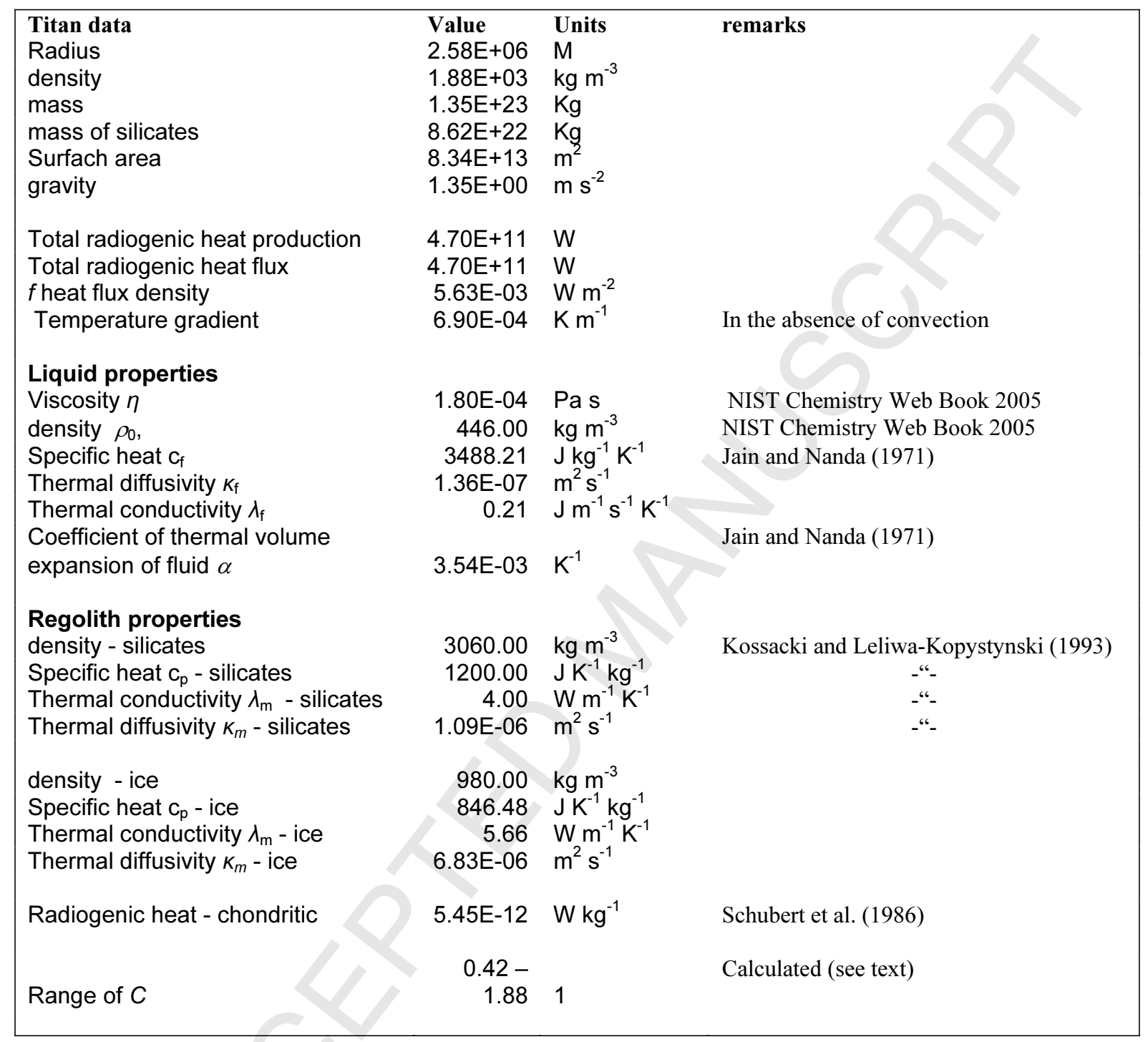


Table 3

Rayleigh number for different permeability $\boldsymbol{k}_{\mathbf{0}}$ and thickness of the regolith $\boldsymbol{h}$. We use the gradient of temperature $\Delta T / h$ and the heat flux density from Table 2 in Eqs. (13) and (14), respectively.

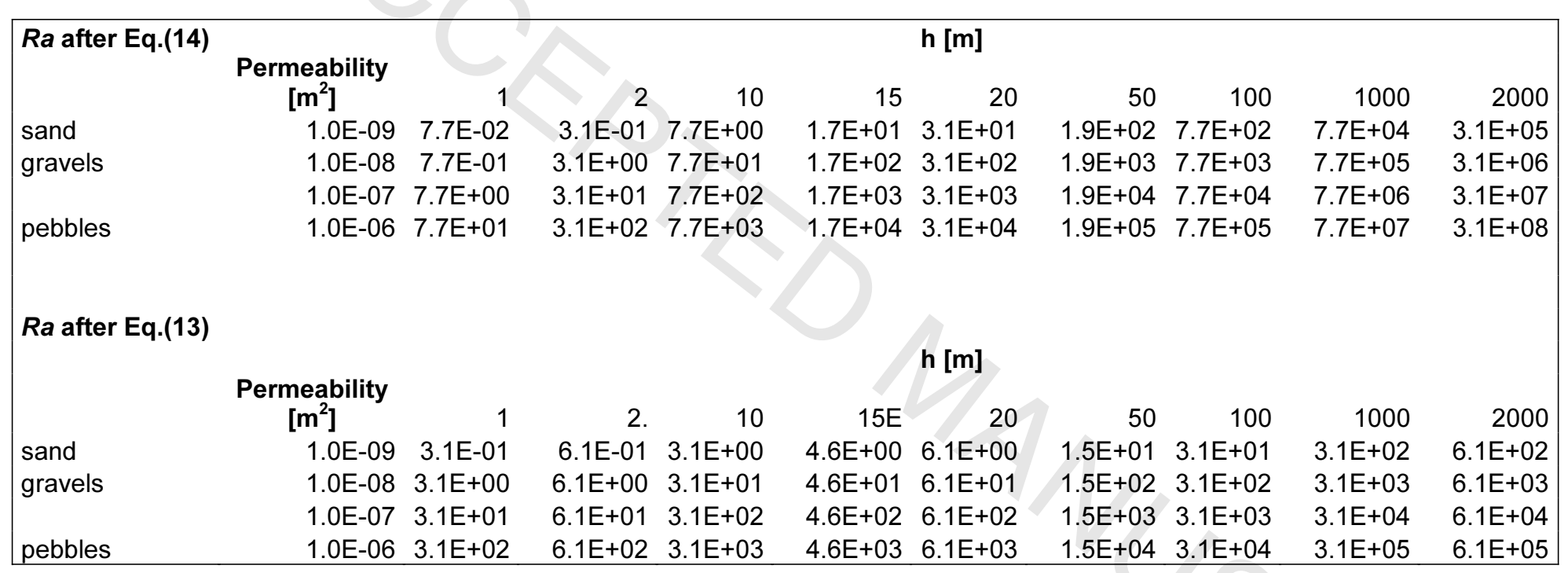


Figure 1 Convection in regolith Czechowski

The considered region and the boundary conditions

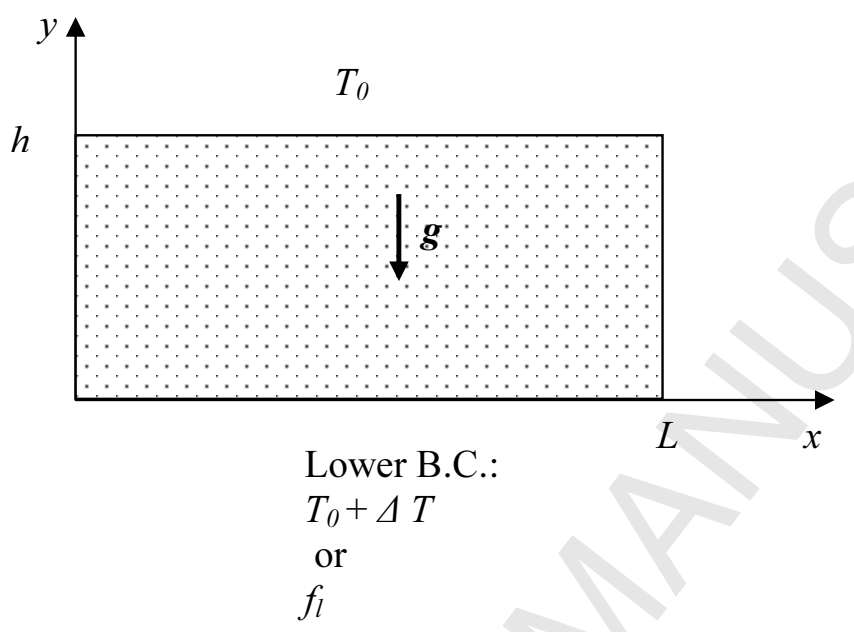


Figure 2 Convection in regolith Czechowski

Comparison of theoretical and numerical $R a^{0}$ for different $L^{\prime}$ and $C=1$. The wavelength of disturbance $l=2 L^{\prime}$.

$$
R a^{0} \text { for } C=1
$$

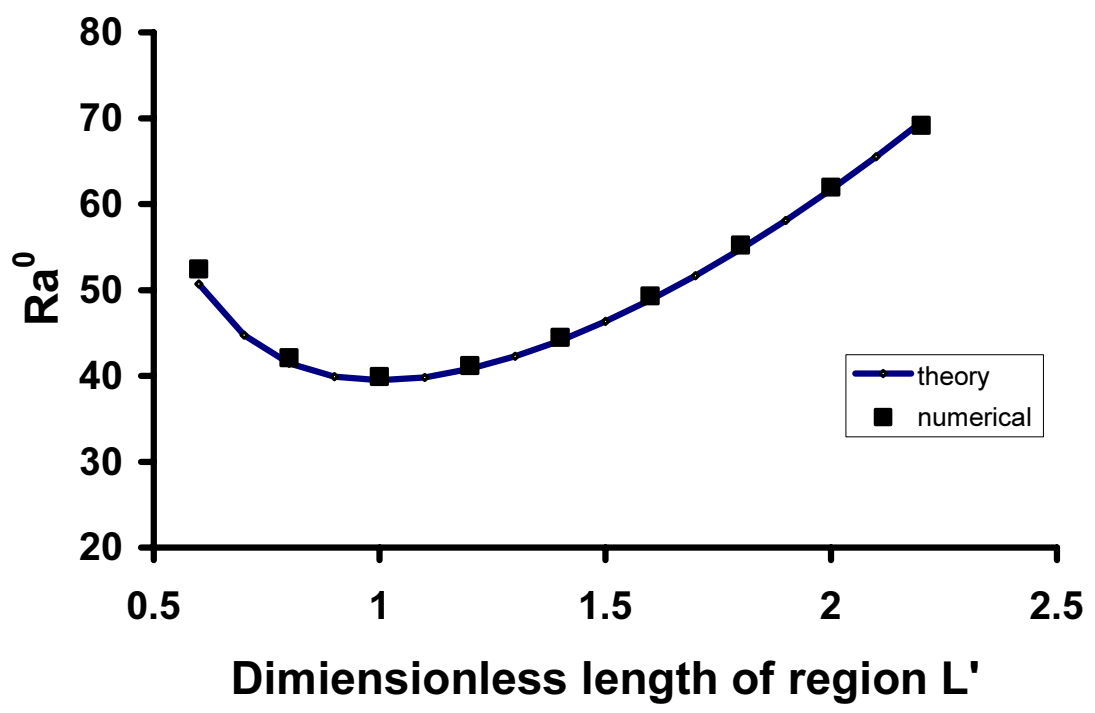


Figure 3. Convection in regolith Czechowski

Ciritcal value of the Rayleigh number $R a_{\mathrm{cr}}$ as a function of $C$ for a few different values of $H_{\mathrm{p}}$, $L^{\prime}=1$, and for $T_{l}=1$ given at the lower boundary.

Racrit (C) for TI given, $L=1, \mathrm{Hp}=0.2,0.4,0.6$

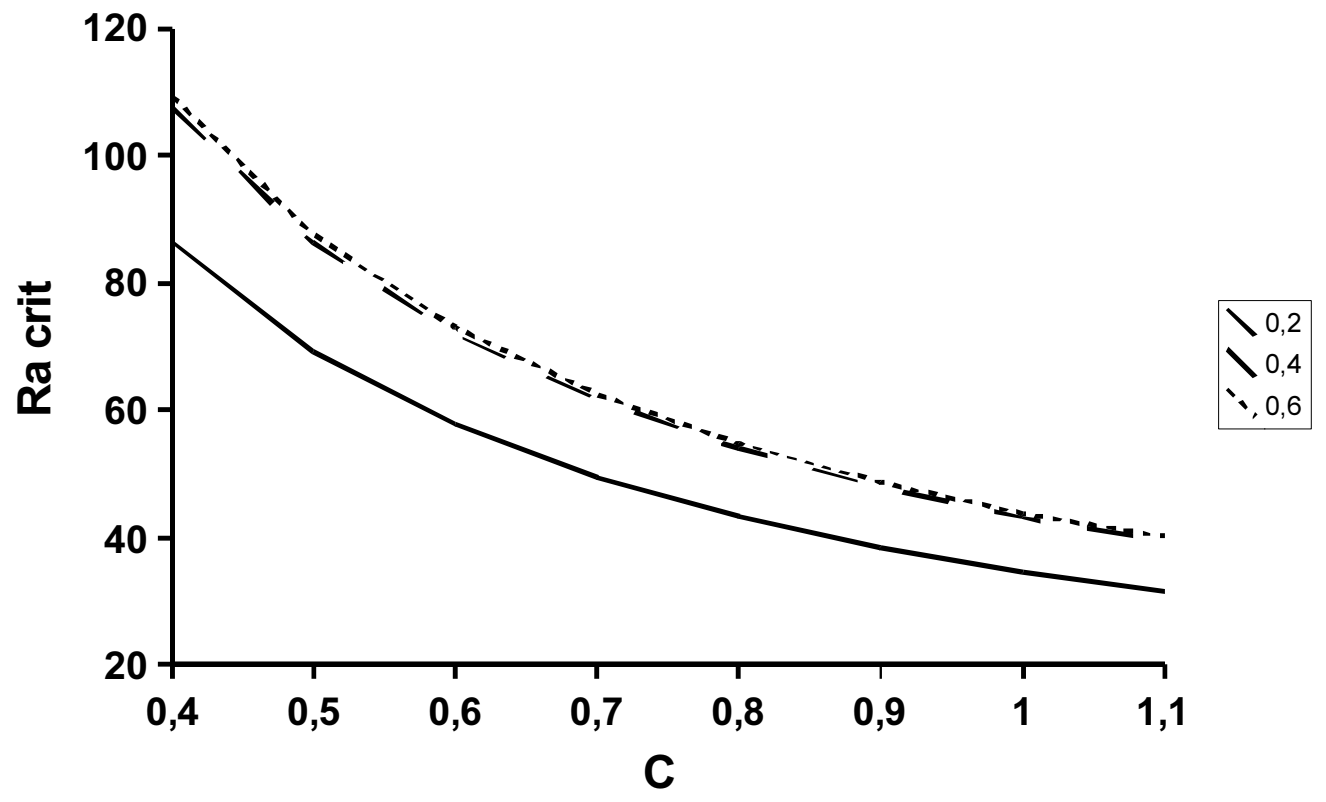




\section{Figure 4 Convection in regolith Czechowski}

Critical value of the Rayleigh number $R a_{\mathrm{cr}}$ as a function of $C$ for $H_{\mathrm{p}}=0.4, L^{\prime}=1$, and for $f_{l}=1$ given at the lower boundary.

Ra crit, fl given, $L^{\prime}=1, H p=0.4$

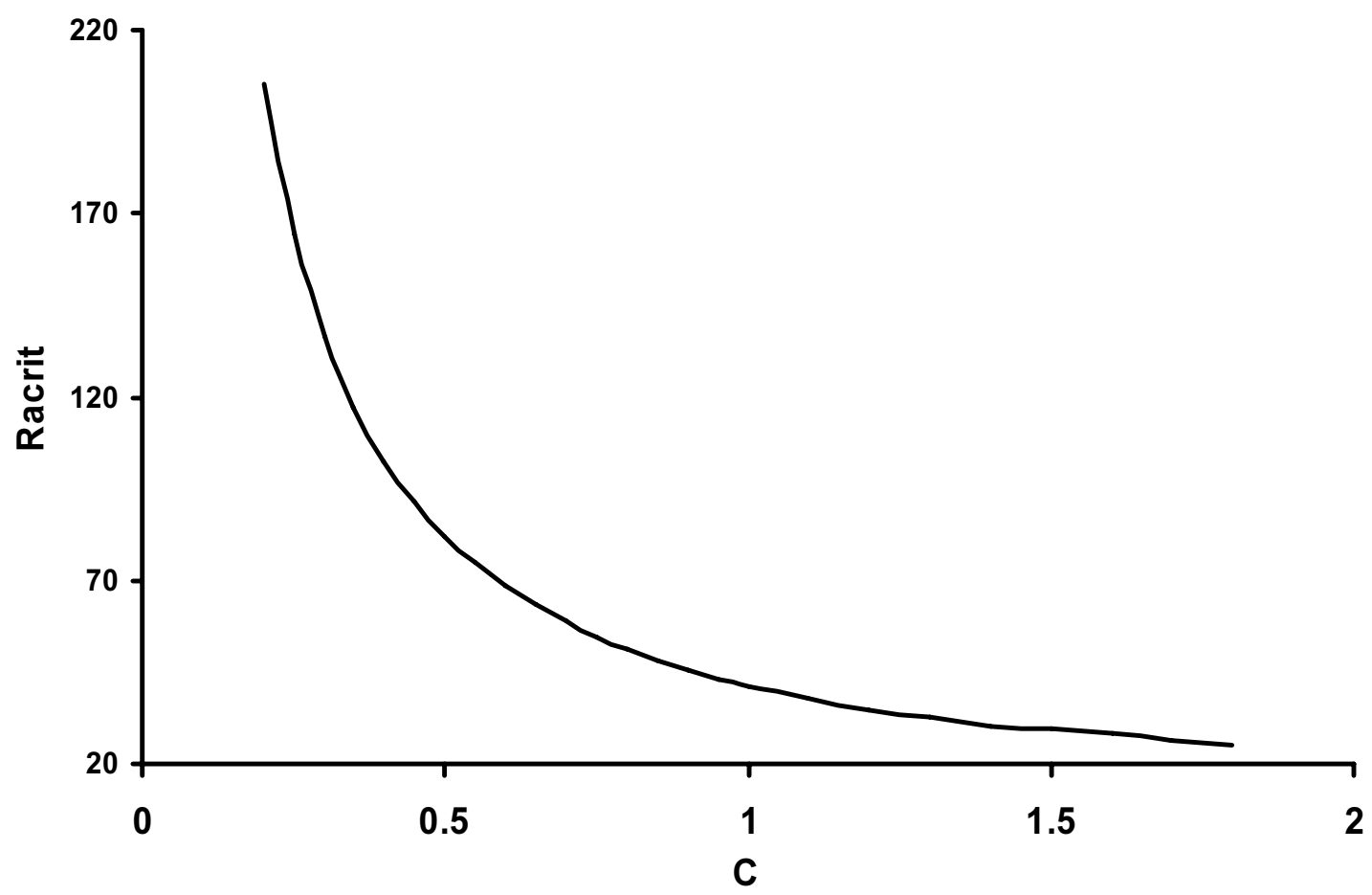


Figure 5 Convection in regolith Czechowski

$R a_{\mathrm{cr}}$ as a function of $H_{p}$ for a few different values of $C . L^{\prime}=1$, and for $f_{l}=1$ given at the lower boundary.

Ra crit $(H p)$ fl given, $L^{\prime}=1, C=0.4,0.8,1.2$

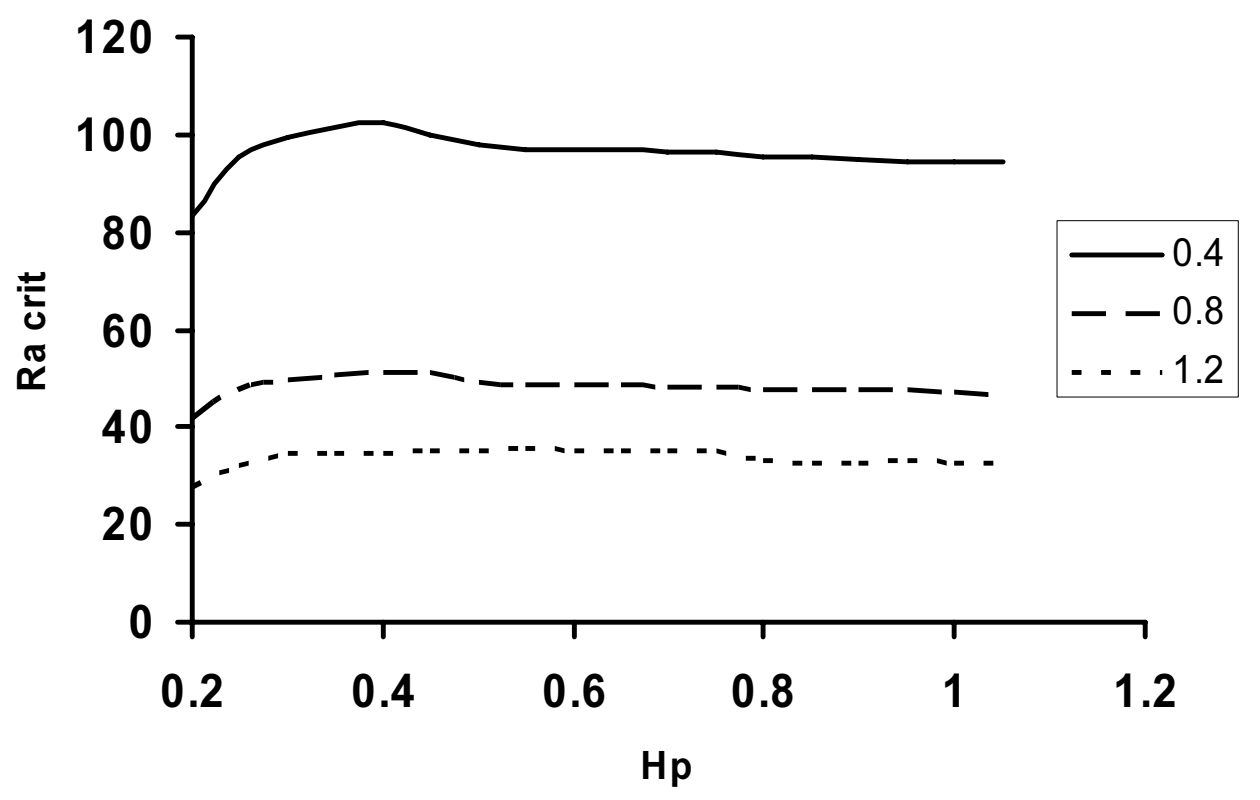


Figure 6 Convection in regolith Czechowski

$R a_{\mathrm{cr}}$ as a function of $H_{p}$ for a few different values of $C . L^{\prime}=1$, and for $T_{l}=1$ given at the lower boundary.

Ra crit(Hp) TI given, $L^{\prime}=1, C=0.4,0.6,0.8,1.1$

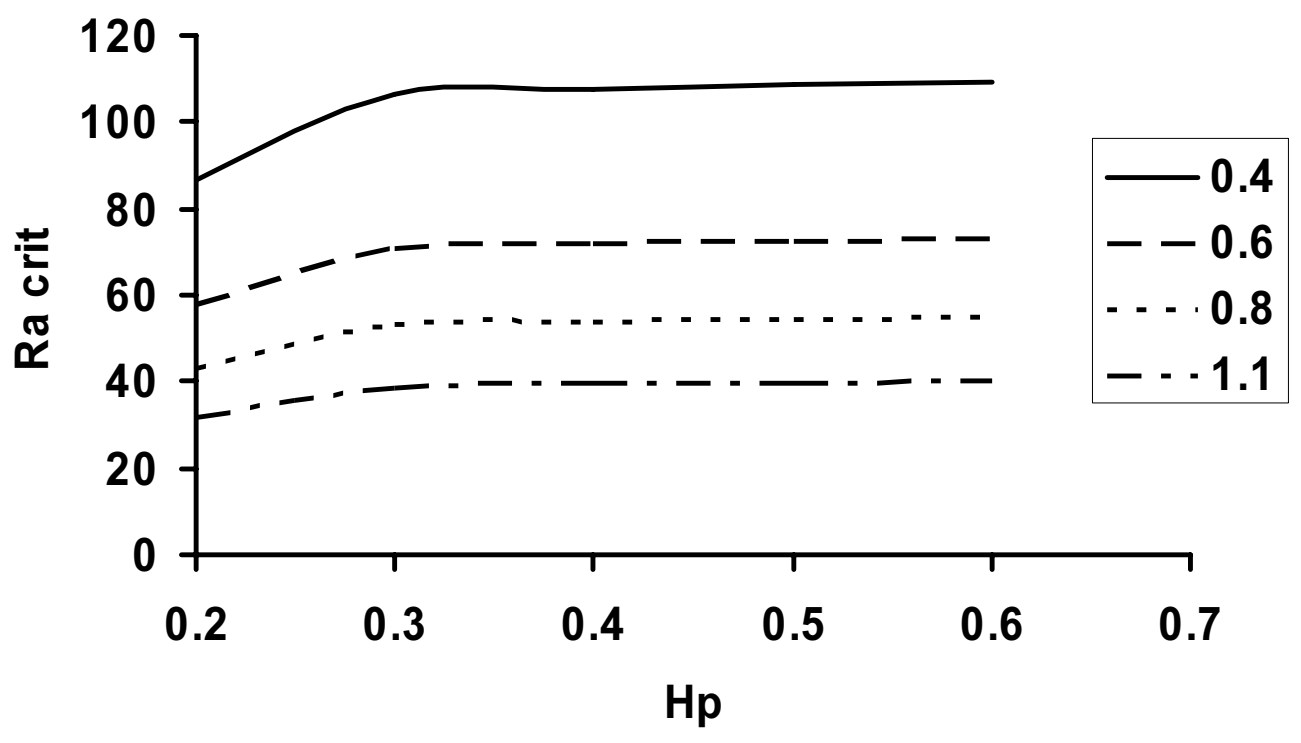


Figure 7 Convection in regolith Czechowski

$R a_{\mathrm{cr}}$ as a function of $L$ ' for a few different values of $C$, for $H_{p}=0.4$, and for temperature $T_{l}=1$ given at the lower boundary.

Ra crit $(\mathrm{L})$ for $\mathrm{TI}$ given, $\mathrm{Hp}=0.4, \mathrm{C}=0.4,0.8,1.2$

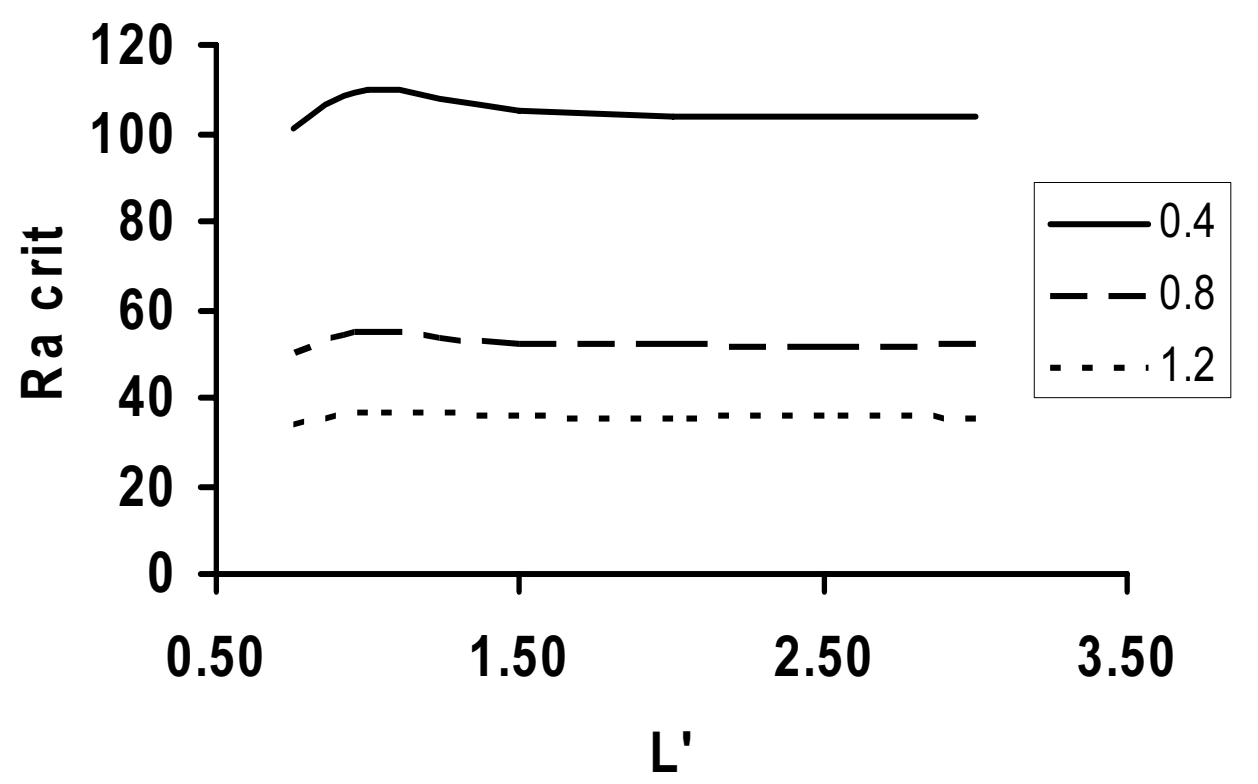


Figure 8 Convection in regolith Czechowski

Rayleigh number $R a(h)$ given by Eq. (14) (upper panel) and Eq. (13) (lower panel) for different values of constant permeability (i.e. for infinite $H_{\mathrm{p}}$ ). The same data are in Table 3 .
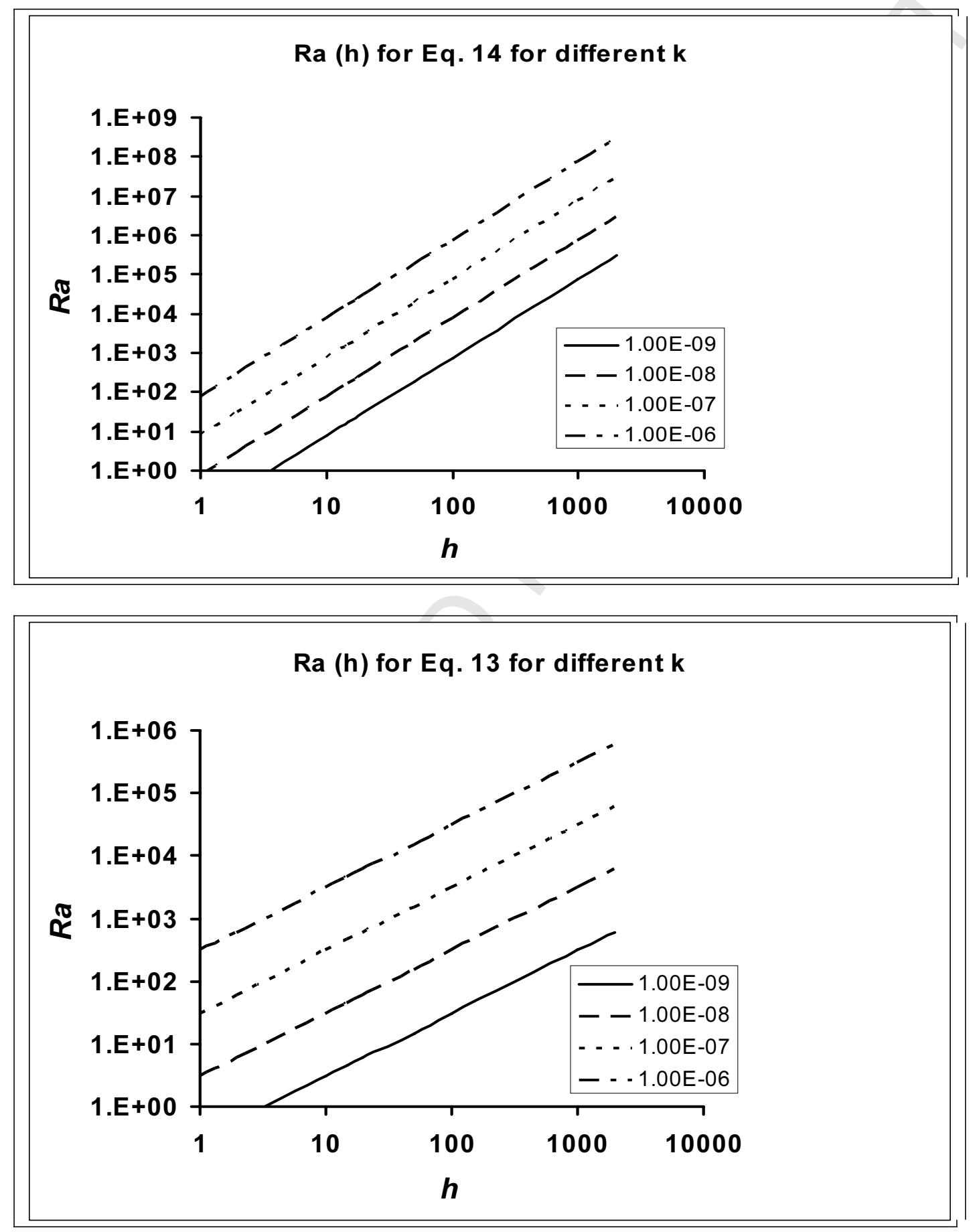